\title{
ANALYSIS OF INTERNAL TIMINGS AND CLOCK RATES OF TERRASAR-X
}

\author{
Ulrich Balss ${ }^{1}$, Helko Breit ${ }^{l}$, Thomas Fritz ${ }^{1}$, Ulrich Steinbrecher ${ }^{2}$, Christoph Gisinger ${ }^{3}$, Michael Eineder ${ }^{1}$ \\ ${ }^{1}$ Remote Sensing Technology Institute (IMF), German Aerospace Center (DLR), \\ D-82230 Oberpfaffenhofen, Germany \\ ${ }^{2}$ Microwave and Radar Institute (HR), German Aerospace Center (DLR), \\ D-82230 Oberpfaffenhofen, Germany \\ ${ }^{3}$ Institute for Astronomical and Physical Geodesy (IAPG), Technische Universität München (TUM), \\ D-80333 Munich, Germany
}

\begin{abstract}
Due to the indirect measurement principle of radar on base of signal travel time, a very precise calibration of the sensor's internal clock with regard to clock rate and potential time offsets relative to the Coordinated Universal Time is an essential prerequisite for accurate pixel localization in Synthetic Aperture Radar. Giving special considerations on this aspect, we developed two algorithms that improve the already high localization accuracy of TerraSAR-X: The either one very precisely determines the true oscillator clock rate, while the other one effectively refines the accuracy of time annotation. Experimental analyses evaluate the obtained improvements in localization accuracy from both techniques.
\end{abstract}

Index Terms - Synthetic aperture radar, TerraSAR-X, precise time measurements, pixel localization accuracy

\section{INTRODUCTION}

The German SAR (Synthetic Aperture Radar) satellite TerraSAR-X (TSX-1) and its twin satellite TanDEM-X (TDX1), launched in June 2007 and June 2010 respectively, provide an unprecedented geometric accuracy. Previous studies showed a ranging accuracy at the centimeter level [1-4]. This accuracy level becomes attainable by a thorough design of all subsystems, including the precise orbit determination [5][6], and the elimination of several processor approximations in the SAR data processing [4].

Radar systems indirectly measure geometric distances by means of the two-way travel time of radar pulses from the radar transmitter to ground and back to the radar receiver. In a focused SAR image, the instant of closest approach of sensor and target as well as the signal travel time at this instant define the two radar time coordinates of azimuth and range. Thus, very precise knowledge of the underlying clock rate and of possible timing offsets is one critical prerequisite for precise position measurements by SAR. Beyond that, as the conversion from travel time to geometric distance usually refers to the vacuum velocity of light, the travel time has to be compensated for all signal propagation delays as we already detailed in our former papers [3][4].

\section{INTERNAL TIMINGS OF TERRASAR-X}

The time annotation of TSX-1 radar data [7] (and synonymously TDX-1 radar data) is based on two independent sources. The more accurate one is the onboard Global Positioning System (GPS) receiver which provides the high-precision pulse per second (PPS) signal [8] and thus the integer number of seconds. The other source is an Ultra Stable Oscillator (USO) on board of TSX-1 which has a significantly higher clock rate but cannot ensure a comparable long-term stability. Strictly speaking, there are two redundant USOs on board (called USO1 and USO2), but only one of both is active while the other one is kept in reserve. In case of TDX-1, the redundant USO2 is active since September 2012.

All internal timings of the SAR instrument are derived from the USO: There are the radar frequency $f_{\text {radar }}$ (about 9.65 Megahertz), the sample rate $f_{\mathrm{ADC}}$ of the Analog to Digital Converter (ADC) whose nominal value is specified by the manufacturer to $f_{\mathrm{ADC}}^{\text {nom }}=329,658,384$ Hertz [7], the configurable Pulse Repetition Interval (PRI) length of the radar pulses and the so-called Instrument Fine Time (IFT). The IFT provides information on the fraction of second based on a counter which is triggered with a clock rate of $f_{\mathrm{ADC}} / 6144$, corresponding to a periodic time $T_{\mathrm{IFT}}$ of about 18.6 microseconds. Every second, the PPS signal resets the counter.

This timing system is designed according to the original pixel localization requirement of 1 meter [9] and is more than sufficient for this purpose. In contrast, when aiming at 1 centimeter localization accuracy, an improved timing procedure is required: On the one hand, the deviation of the true ADC sample rate $f_{\mathrm{ADC}}^{\text {true }}$ from the nominal value $f_{\mathrm{ADC}}^{\text {nom }}$ has to be considered. On the other hand, the quantization error of the IFT significantly contributes to the overall azimuth error. Considering the typical ground track velocity of TerraSAR- 
$\mathrm{X}$ which amounts to about 7050 meters per second, the ground track moves by approximately 13 centimeters during one 18.6 microseconds period of the trigger signal. Assuming the quantization error is uniformly distributed, the expected standard deviation of this error contribution amounts to 3.8 centimeters. However, the trigger rate is predetermined by the hardware and therefore it is not configurable.

In the following two sections, a method to determine the true ADC sample rate and an algorithm which significantly alleviates the effect of the limited annotation accuracy of the IFT are presented. As the ADC sample rate is not very volatile, it suffices to occasionally evaluate its value in the context of instrument recalibration. In contrast, the IFT refinement has to be individually applied for each single datatake at the beginning of SAR data processing.

\section{EVALUATION OF THE INTERNAL CLOCK RATE}

Since all USO-derived frequencies are proportional to the USO clock rate and all USO-derived time intervals are reciprocally proportional to it, we propose to indirectly determine $f_{\mathrm{ADC}}^{\text {true }}$ by the comparative measurement of a long time interval which may be the duration of a long datatake. Based on $f_{\mathrm{ADC}}^{\text {nom }}$ as an initial guess for $f_{\mathrm{ADC}}$, we estimate the datatake duration by summing up the individual USO-derived lengths of all PRIs annotated in the datatake. This estimate is compared with an external reference: the accurate datatake duration that we get from the difference of the PPS-derived GPS time tags of datatake start and stop time. The discrepancy between USO-based estimate and accurate PPS-based duration yields a measure for the deviation of our initial guess $f_{\mathrm{ADC}}^{\text {nom }}$ from $f_{\mathrm{ADC}}^{\text {true }}$. This is the basic idea of our approach. In fact, datatake start and stop time generally do not exactly coincide with the instants of PPS pulses. Therefore both time tags, datatake start and stop time, consist of a PPS-derived integer part and an USO-derived fractional part. However, the following deductions show that this property does not affect the solvability of the arithmetic problem.

Let $1+\alpha$ be the ratio of a-priori unknown $f_{\mathrm{ADC}}^{\text {true }}$ and the given $f_{\mathrm{ADC}}^{\mathrm{nom}}$ so that

$$
f_{\mathrm{ADC}}^{\text {true }}=(1+\alpha) \cdot f_{\mathrm{ADC}}^{\mathrm{nom}} .
$$

As soon as $(1+\alpha)$ is determined, it can be used as correction factor for all USO derived frequencies while $(1+\alpha)^{-1}$ yields a correction factor for all USO derived time intervals. Consequently, the true datatake duration $\Delta t$ that we measured on base of the time tags of datatake start and stop, is given by

$$
\Delta t=\Delta t_{\mathrm{GPS}}+\Delta t_{\mathrm{IFT}}^{\mathrm{nom}} \cdot(1+\alpha)^{-1}
$$

where $\Delta t_{\mathrm{GPS}}$ is the PPS-based difference in integer seconds while $\Delta t_{\mathrm{IFT}}^{\mathrm{nom}}$ denotes the difference in the IFT-based fraction of seconds. The superscript "nom" indicates that $\Delta t_{\mathrm{IFT}}^{\mathrm{nom}}$ is computed on base of $f_{\mathrm{ADC}}^{\text {nom }}$.

Let $\Delta t_{\mathrm{PRI}}^{\mathrm{n} \text { nom }}$ be our estimate for the datatake duration that we obtain by summing up the PRI-lengths on base of $f_{\mathrm{ADC}}^{\text {nom }}$. Since $\Delta t_{\mathrm{PRI}}^{\mathrm{nom}}$ is an USO-derived time interval, we have to correct it by factor $(1+\alpha)^{-1}$ if we aim to get the true duration

$$
\Delta t=\Delta t_{\mathrm{PRI}}^{\mathrm{nom}} \cdot(1+\alpha)^{-1} .
$$

Equations (2) and (3) can be equated and solved for

$$
\alpha=\frac{\Delta t_{\mathrm{PRI}}^{\mathrm{nom}}-\Delta t_{\mathrm{IFT}}^{\mathrm{nom}}}{\Delta t_{\mathrm{GPS}}}-1 \text {. }
$$

By inserting equation (4) in equation (1), we finally get

$$
f_{\mathrm{ADC}}^{\text {true }}=\frac{\Delta t_{\mathrm{PRI}}^{\mathrm{nom}}-\Delta t_{\mathrm{IFT}}^{\mathrm{nom}}}{\Delta t_{\mathrm{GPS}}} \cdot f_{\mathrm{ADC}}^{\text {nom }} .
$$

Since the radar frequency as well as the ADC sample rate is derived from the USO, our method also provides its true value $f_{\text {radar }}^{\text {true }}$ if we convert $f_{\mathrm{ADC}}^{\text {true }}$ from equation (5) by using the knowledge of the underlying frequency dividers.

From a detailed error analysis, we learned that the accuracy of the obtained results depends on the ratio of the given IFT clock rate, which governs the resolution of the values in the numerator of equation (5), and the datatake duration (in the denominator). This is the reason why long datatakes are of advantage for the precise determination of $f_{\mathrm{ADC}}^{\text {true }}$ (although the IFT itself can be further refined as we show later). Averaging the individual results from several independent measurements based on several datatakes of different length is another strategy to improve the obtainable accuracy.

The used datatakes might be selected from operational Stripmap or ScanSAR datatakes of the TerraSAR-X and TanDEM-X missions. However, there are thermal constraints which limit the maximum duration of imaging datatakes. Primarily, they are due to the warming of the SAR instrument during the radar pulse transmission. In contrast, as we are not interested in the image content of the datatake but solely in the datatake annotation, receive-only system datatakes, especially designed with regard to the application on hand, are a mean to overcome these constraints.

In August 2013, TSX-1 and at exactly the same time TDX-1, each recorded 14 system datatakes. The datatake lengths amount to about 11.5 minutes. On base of these datatakes, we measured $f_{\mathrm{ADC}}^{\text {true }}=329,658,361.0 \pm 0.2$ Hertz for USO1 of TSX-1 and $f_{\mathrm{ADC}}^{\text {true }}=329,658,345.5 \pm 0.3$ Hertz for USO2 of TDX-1. Thus, the previously used nominal value overestimated the true ADC sample rate by about $23 \mathrm{Hertz}$ in case of TSX-1 and about 38 Hertz in case of TDX-1.

The difference in obtained $f_{\mathrm{ADC}}^{\text {true }}$ between TSX-1 and TDX-1 can be cross-check by another, independent measurement method. Driven by the bistatic TanDEM-X mission, where both satellites are involved, and synchronization information between the satellites' oscillators is required in order to compensate for phase artifacts in bistatic SAR processing, TSX-1 and TDX-1 have a direct horn antenna link. With it so-called synchronization pulses can be exchanged [10][11], interleaving the usual datatake acquisition. The 
received signals are recorded to be analyzed on ground [12]. We also made use of this opportunity for our TSX-1/TDX-1 pairs of system datatakes. Here, the synchronization pulse analysis led to a frequency difference of $\Delta f_{\mathrm{ADC}}^{\text {true }}=15.4 \pm 0.1$ Hertz between TSX-1 and TDX-1 and confirms in so far the results of our absolute measurement where the measured frequencies differ by $\Delta f_{\mathrm{ADC}}^{\text {true }}=15.5 \pm 0.4$. However in contrast to our approach, the synchronization pulse measurements provide no information on the absolute frequencies.

\section{REFINEMENT OF THE AZIMUTH TIMING}

The acquisition time of every TerraSAR- $\mathrm{X}$ radar echo line is annotated in the instrument source packet (ISP) header of the echo line on base of integer GPS seconds and the USObased IFT for the leading edge of the respective PRI. For this reason, the knowledge, when the echo was recorded, is determined by the annotation accuracy of the IFT. On the other hand, the relative time period between two echo lines is given very precisely by the PRI length. Thus, our approach to alleviate the effect of IFT quantization is to link the coarse absolute time information from subsequent ISPs on base of the precisely known time interval between them.

Figure 1 shall illustrate the basic idea of our algorithm. The sketch outlines the available timing information from the subsequent ISP headers. Starting with the time annotation of the first ISP, we know that the leading edge of its echo data was recorded at a point within a time interval of 18.6 microseconds, symbolized in the sketch by the topmost medium grey box. Based on the time annotation of the second ISP (next medium grey box one row below) and the precisely known PRI length, we can calculate back to the first ISP and get another time interval (light grey box) as a second, independent measurement for its acquisition time. In general, both intervals do not coincide but they shall intersect. The sole consistent implication from both observations is that the exact acquisition time of the first ISP must be within the intersection of both time intervals. Otherwise, the IFT count in one of both ISP headers had to be by one step higher or lower. Continuing this procedure based on the time annotation from the third ISP and so on, we get further independent measurements which can narrow the uncertainty of the acquisition time of the first radar echo. In this way, we end-up at a much smaller time interval, symbolized in the sketch by the leftmost dark grey box. In the final step of our algorithm, the precise PRI lengths give us an instrument to reversely iterate from the refined acquisition time of the first echo line to the precise acquisition times of all other echo lines, like it is sketched in the last row of Figure 1.

Two constraints define the usable number of ISPs for our algorithm and as a consequence the obtainable timing accuracy. As IFT and PRI length are likewise derived from the USO, they have a least common multiple, and after their phasing pattern recurs so that there is only redundant infor-

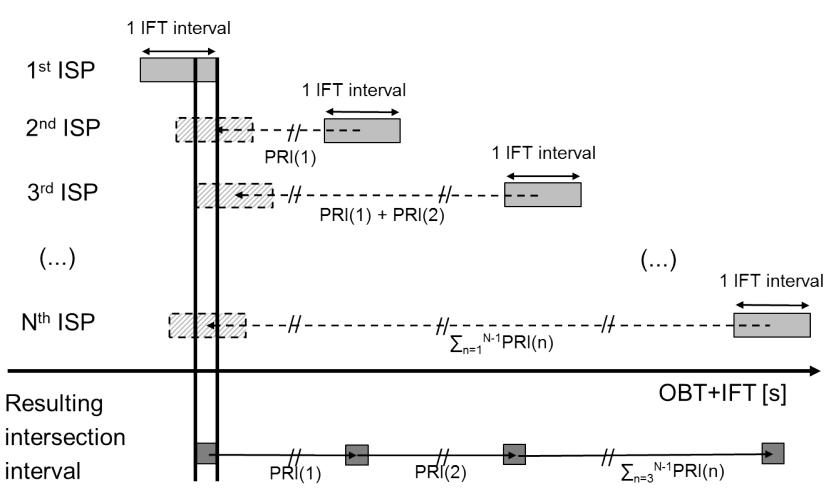

Figure 1: Sketch (not to scale) of the basic idea of our IFT refinement approach

mation. Depending how coprime both periods are, ISPs from the same GPS second provide at best an uncertainty interval for the refined azimuth time of 84 nanoseconds, providing that the phasing of the IFT trigger signal relative to the PPS signal, that resets the IFT counter, was known. In fact, it is a-priori unknown, and for this reason we have to set-up our algorithm for every GPS second. Consequently, we determined that the results of these independent refinement runs typically coincide within $T_{\mathrm{IFT}} / 32$, which yields an estimate for the uncertainty in the phasing between both signals.

\section{EXPERIMENTAL VERIFICATION}

The pixel localization accuracy of a SAR system can be verified by comparing the range and azimuth times of corner reflectors (CRs) in focused SAR images with their expected values obtained from precise terrestrial geodetic measurements of their positions and taking into account signal propagation delays. The measurement series on hand is based on a CR with very precisely known ground coordinates (better than 5 millimeters) which we installed near the geodetic observatory Wettzell, Germany [3][4]. Up to now, 52 high resolution Spotlight (HS) datatakes were recorded in two different acquisition geometries.

In order to evaluate the gain from the here presented algorithms, we investigated 3 different variants of SAR data processing. We focused the Wettzell datatakes by each of them and compared the obtained localization accuracy of our CR. The first, for quite some time outdated processing variant used none of both innovations. In the second variant, $f_{\mathrm{ADC}}^{\text {true }}$ replaces $f_{\mathrm{ADC}}^{\mathrm{nom}}$. Until recently, this was the operationally employed TerraSAR-X SAR processor. The third, current variant additionally includes the refinement of the IFT.

Figure 2 shows the scatter plots of the measured location offset. Table 1 gives a statistical analysis of the obtained results. The first processing variant led to a distinct range bias of several centimeters. As $f_{\mathrm{ADC}}^{\text {nom }}$ overestimates the ADC sample rate, it causes an inversely rated underestimation of signal travel time and, as a consequence, range distances. 

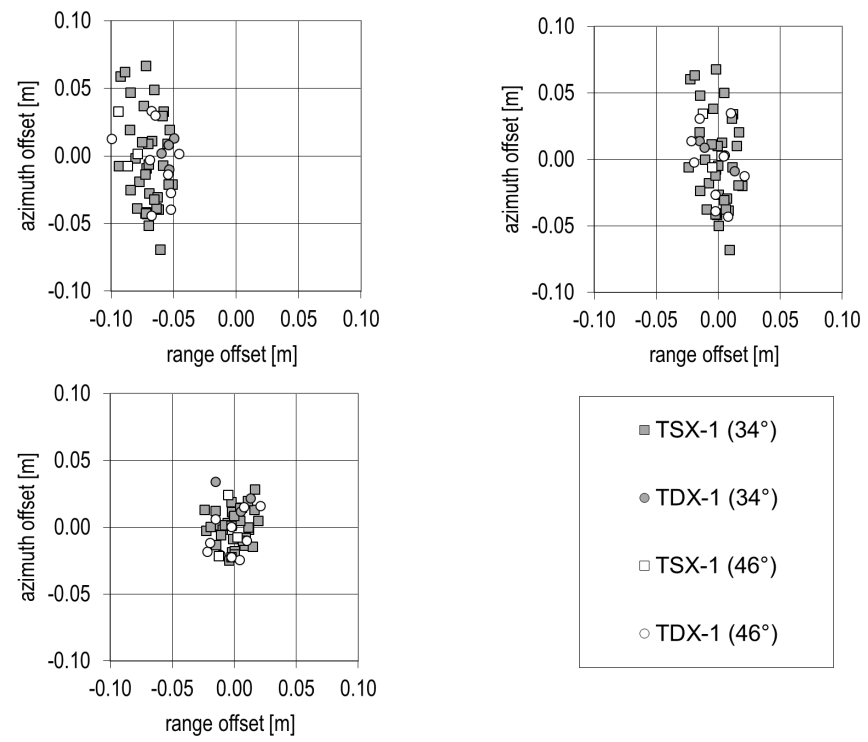

Figure 2: Obtained pixel localization for the different processing variants in investigation: top left: old approach. top right: until recently used approach. bottom left: current approach.

Table 1: Statistical analysis of the obtained localization offset

\begin{tabular}{|l|r|r|}
\hline processing variant & $\begin{array}{r}\text { azimuth position } \\
\text { offset [mm] }\end{array}$ & $\begin{array}{r}\text { range position } \\
\text { offset [mm] }\end{array}$ \\
\hline 1. old & $-3.6 \pm 32.1$ & $-68.8 \pm 12.9$ \\
\hline 2. until recently & $-2.3 \pm 32.1$ & $-1.0 \pm 11.4$ \\
\hline 3. current & $0.0 \pm 15.0$ & $0.0 \pm 11.5$ \\
\hline
\end{tabular}

Azimuth distances shall be underestimated at the same rate, but within 5 kilometers azimuth extent of a HS scene the effect is hardly detectable. The usage of $f_{\mathrm{ADC}}^{\text {true }}$ in the second variant overcomes the underlying problem so that the range bias disappears. There remains the quantization error of the coarsely annotated IFT which still dominates the overall location error in azimuth. The IFT refinement in the third variant effectively reduces this error contribution to the extent that the overall azimuth error is approximately halved.

\section{CONCLUSIONS}

Considering the essential significance of precise time measurements for SAR, the pixel localization accuracy significantly profits from the presented algorithms. The measured $f_{\mathrm{ADC}}^{\text {true }}$ is already included in the operational instrument calibration of TSX-1 and TDX-1. Henceforth, it shall be monitored at routine intervals based on specific receive-only system datatakes. The likewise presented IFT refinement scheme is a new feature in the new version 4.10 of the TerraSAR-X Multimode SAR Processor (TMSP) which got operational May, 22, 2014. For the analysis of the residual location error, which remains after these progresses, we conclude a major contribution in the limited observability of the exact phasing between IFT trigger signal and PPS signal.

\section{ACKNOWLEDGMENTS}

The work was partially funded by the German Helmholtz Association HGF through its DLR@Uni Munich Aerospace project "Hochauflösende geodätische Erdbeobachtung". We thank the Federal Agency for Cartography and Geodesy (BKG) for their kind allowance to install our corner reflectors at their property in Wettzell and for their local support.

\section{REFERENCES}

[1] A. Schubert, M. Jehle, D. Small, and E. Meier, "Mitigation of Atmosphere Perturbations and Solid Earth Movements in a TerraSAR-X Time-Series," Journal of Geodesy, Online First, September 23, 2011.

[2] M. Eineder, C. Minet, P. Steigenberger, X.Y. Cong, and T. Fritz, "Imaging Geodesy - Toward Centimeter-Level Ranging Accuracy with TerraSAR-X," IEEE Trans. on Geosci. and Remote Sens., vol. 49, no. 2, pp. 661-671, 2011.

[3] U. Balss, X.Y. Cong, R. Brcic, M. Rexer, C. Minet, H. Breit, M. Eineder, and T. Fritz, "High Precision Measurement on the Absolute Localization Accuracy of TerraSAR-X," Proc. IGARSS 2012, Munich, pp. 1625-1628, 2012.

[4] U. Balss, C. Gisinger, X.Y. Cong, R. Brcic, P. Steigenberger, M. Eineder, R. Pail, and U. Hugentobler, "High Resolution Geodetic Earth Observation with TerraSAR-X: Correction Schemes and Validation," Proc. IGARSS 2013, Melbourne, pp. 4499-4502, 2013.

[5] O. Montenbruck, M. Wermuth, and R. Kahle, "GPS Based Relative Navigation for the TanDEM-X Mission", ION-GNSS, Portland, 2010.

[6] Y. Yoon, M. Eineder, N. Yague-Martinez, and O. Montenbruck, "Precise Trajectory Estimation and Quality Assessment", IEEE Trans. on Geosci. and Remote Sens., Vol. 47, No. 6, pp. 1859-1868, 2009.

[7] Astrium GmbH, Annex 3.5: TerraSAR-X Data, TX-AED-DD0001, 2003 (unpublished).

[8] Hofmann-Wellenhof, B., H. Lichtenegger, and E. Wasle, GNSS Global Navigation Satellite Systems, Springer, Wien, 2008.

[9] Fritz, T., and M. Eineder, TerraSAR-X Ground Segment Basic Product Specification Document, TX-GS-DD-3302, v1.5, 2008.

[10] M. Eineder, "Oscillator Clock Drift Compensation in Bistatic Interferometric SAR,” Proc. IGARSS 2003, Toulouse, 2003.

[11] M. Younis, R. Metzig, and G. Krieger, "Performance Prediction of a Phase Synchronization Link for Bistatic SAR," IEEE Geosci. and Remote Sens. Letters, vol. 3, no. 3, pp. 429-433, 2006.

[12] H. Breit, T. Fritz, U. Balss, A. Niedermeier, M. Eineder, N. Yague-Martinez, and C. Rossi, "Processing of Bistatic TanDEM-X Data," Proc. IGARSS 2010, Vancouver, 2010. 